\title{
La Revista basurero
}

\author{
The Journal landfill
}

\section{Leonardo Romero}

\begin{abstract}
Facultad de Ciencias Biologicas, Universidad Nacional Mayor de San Marcos. Ciudad Universitaria de San Marcos Av. Venezuela s/n. Apartado postal 110058, Lima 11, Perú.

Email Leonardo Romero:

Iromeroc@unmsm.edu.pe
\end{abstract}

\section{Aumenta la investigación y las publicaciones científicas con todos sus males}

El interés por publicar el resultado de nuestras investigaciones es inherente a la investigación, investigamos para descubrir la verdad y comunicar lo que descubrimos. Sin embargo, al igual que un ente vivo es modelado por las circunstancias o el ambiente en que vive, la investigación científica y sus publicaciones evolucionan como consecuencia de cambios sociales, económicos y culturales. Los cambios sociales y en investigación son tangibles y pueden sintetizarse si se compara la información global para el ańo 2002 con el $2007^{1}$; el gasto global en investigación prácticamente se duplicó de 790,3 billones en el 2002 a 1145,7 en el 2007, el número de investigadores se incrementó de 5,7 millones a 7,1; el número de publicaciones científicas aumentó de 1,09 millones en el 2002 a 1,94 en el 2010, es decir en un $77 \%{ }^{1}$. En estas diferencias, fueron las economías emergentes como China, Brasil e India las que en conjunto aumentaron sus inversiones en ciencia, tecnología y desarrollo de 17 a $24 \%$.

Aunque el Perú todavía no figura en las estadísticas y cifras generales de desarrollo de investigación científica a nivel mundial, toda una jerga sobre ciencia, tecnología, desarrollo e innovación se escuchan en los pasadizos de nuestros institutos y centros académicos, y responden a las intenciones de remontar nuestro actual nivel científico (por lo menos el nivel en que nos colocan bibliométricamente ${ }^{2}$ ), dar a conocer nuestra producción en investigación científica, y cómo ella puede ser la fuente de desarrollo del país. Para instituciones como la Universidad Nacional Mayor de San Marcos (UNMSM) y otras universidades públicas peruanas acreditar una consistente y robusta actividad científica se convierte en un objetivo estratégico para deslindar nuestra labor universitaria con la de otras instituciones semejantes en la cuales el lucro es una de sus finalidades principales. Para la UNMSM, la acreditación de su labor académica y de investigación, y el mostrar la calidad de su producción intelectual y científica serán los pilares que permitirán a la UNMSM tener el peso necesario en las decisiones que involucren el desarrollo nacional. Desde el año 1998, en la UNMSM se promovieron las revistas de investigación, dentro del marco del reglamento de investigaciones y como una herramienta para facilitar la difusión de los resultados de las investigaciones en las diferentes facultades. La Revista Peruana de Biología es una de esas revistas, pero que además asumió el reto de convertirse en un medio de excelencia para la publicación de los trabajos principalmente en las áreas de biodiversidad y biotecnología en el Perú. A pesar del tiempo transcurrido, su edición aún es inestable, generalmente producido por problemas económicos, organizacionales y logísticos, causando la dilación en el tiempo de entrega como uno de sus problemas más importantes. A estos problemas se suman otros relacionados al quehacer de cualquier revista científica incluso las del main stream, el problema de las malas conductas y fraudes. Dentro del contexto de calidad que la UNMSM busca, el incremento de trabajos fraudulentos se torna un fenómeno delicado y a su vez demanda acciones más enérgicas por las consecuencias que pueden tener. Pero, rechazar un trabajo fraudulento una vez detectado no es el problema, sino la agobiante pregunta ¿Por qué nos llegan tantos?

\section{¿Qué no harían por publicar?}

La publicación de un artículo de investigación científica respalda la labor realizada, es uno de los productos más importantes de las instituciones dedicadas a la ciencia y las labores académicas y universitarias, crea y desarrolla el prestigio del investigador y de la institución ante la sociedad; todo esto acarrea como consecuencia fondos para investigación, bienestar, crecimiento y desarrollo para el investigador y la institución (hechos no necesariamente vinculantes). Por otro lado, un editor es un espectador y actor del "drama de la publicación científica en el escenario del desarrollo de la ciencia”. Publicar un trabajo científico no es un tema sencillo y podría en algunos momentos ser una actividad que se envuelva en una vorágine de intereses de todo tipo, de colores, sabores, y malos olores. Uno de esos momentos comienza cuando llega a la edición un trabajo con síntomas de malas conductas o fraudes. Aquí se enfrenta la intención del autor con la misión del editor que es de difundir información de calidad y que satisfaga a la comunidad científica y a la sociedad. Aunque estos malos comportamientos y fraudes no son causados por la revista o el editor, sino provienen de la comunidad científica misma, es el editor el que se bate con ellos ${ }^{3}$, aunque ponga en riesgo su salud y su vida.

Para el Council of Science Editors ${ }^{4}$ la mala conducta en investigación se refiere a malos tratamientos de los sujetos de investigación (personas o animales), la falsificación y fabricación de datos y la piratería y plagiarismo. Pero en otros ámbitos se separan los fraudes de las malas conductas por aspectos volitivos y veniales. Mientras que, las malas conductas generalmente son cometidas sin "intensión”, y pueden ser relativas a errores metodológicos (diseńos experimentales, incumplimiento de normas y recomendaciones), procedimientos “culturales" (como la falta de ética en autorías, las publicaciones en salchichón); tenemos por otra parte los fraudes, en los cuales la intensión del autor es 
conscientemente expresa o velada pero innegable (falsear datos, inventarlos, ocultar información, "cocinar datos", plagio, etc.).

\section{El lado oscuro de la publicación científica}

Oscuro porque el editor generalmente desconoce quién es el autor y su trabajo, y ellos deben ser escudrińados en su intensión de publicar y en la veracidad de su información. Es decir en esta primera escena de la revisión, los editores no sabremos de dónde vendrá el ataque o cómo vendrá; en esa oscuridad se reciben los trabajos. Aunque existen sistemas y software ${ }^{5}$ para detectar el plagiarismo y otras malas conductas y fraudes, estos son un primer tamiz en este aspecto. Sin embargo, varios editores consideran a la primera revisión del trabajo como la parte del proceso en que se cuentan con menos herramientas, personal adecuado y tiempo para detectarlas ${ }^{6}$. Aunque parezcan demasiadas precauciones, casi lindando con algún estado mental desquiciado, no estamos muy lejos de las grandes editoras como Elsevier ${ }^{7}$ que ponen mucho énfasis en adiestrar a sus editores y autores sobre estos temas, los aspectos legales para su incumplimiento y las consecuencias de la infracción.

\section{La alegría de ser coautor sin responsabilidad}

En este drama identificar las malas conductas y fraudes es uno de los papeles del editor y de los revisores (peer review). Sin embargo, los actuales modos de investigación interdisciplinarios, las investigaciones en grandes laboratorios, y donde es frecuente que se produzcan artículos con muchos autores, crean una escena en la que el responsable del artículo puede cargar con la culpa de la mala conducta y fraude ${ }^{8}$ (en muchos casos con sanciones de parte de su institución) y los otros autores (mal denominados, pero convenientemente coautores) no sufren más sanciones que la vergüenza. Y la pregunta es donde surgió el "problema”, ¿no hubo discusión entre los autores? ¿No se compartieron datos entre autores? ¿Cómo interactuaron? ¿Cómo se hizo el trabajo? ¿Todos no tienen las mismas responsabilidades? Este aspecto nos lleva a la conclusión de que las instituciones, y no solamente los miembros de los comités editoriales, deben asumir e identificarse con las buenas prácticas de investigación y buena conducta, de manera que la conducta fraudulenta pueda ser por lo menos identificada y aislada9. La participación de todos los autores en la presentación del trabajo, la posibilidad de contactarse con cada uno de los autores, cartas de compromiso y otros, son filtros que están dirigidos a identificar una posible mala conducta.

\section{El peer review como filtro}

El revisor de un trabajo que pretende ser publicado es un par (o igual) o muchas veces otro científico con más experiencia en el tema y que puede dar luces como mejorar la presentación de la información. El revisor (peer review) es una instancia presente en la edición científica casi desde los inicios de la publicación científica hace más de 300 años. Una encuesta realizada a autores de revistas de alto factor de impacto (FI) en el año $2008^{10}$ a pedido del Publishing Research Consortium, mostró que el 43\% de los encuestados consideraba que el peer review filtra los trabajos fraudulentos y con malas conductas. Ante esta percepción se enfrentan investigaciones que ponen en tela de juicio la severidad y consistencia del peer review para detectar casos de fraude ${ }^{11}$. Los revisores juegan un papel importante en la determinación de la calidad del trabajo, la pertinencia y la actualidad, por su experiencia son los que están en mejores condiciones de detectar mala conducta. Sin embargo, la fabricación o invención de resultados es más difícil de detectar sobre todo en determinados temas en los cuales se arguye la imposibilidad de la repetición del experimento. Pero en el área de biodiversidad, detectar especies que no existen en el lugar, o especies inventadas es más fácil por la experticia de los revisores. Por lo tanto el revisor es un filtro fino, dedicado a ciertos tipos de malos comportamientos y seleccionar trabajos de calidad y con información relevante.

\section{La retracción, la punta del iceberg}

Los niveles en que el plagiarismo y las malas conductas son muy altos. En el 2010 la editora Francis \& Taylor del grupo Nature Publishing escudrińo los trabajos recibidos en los últimos meses y en una de sus revista se detectó el 23\% de los trabajos con algún grado de plagiarismo ${ }^{12}$. Recientemente Daniele Fanelli analizando los artículos que solo han perpetrado fraudes como falsificación, fabricación de datos, alteración de información, etc. encuentra que casi el 2\% admite haber cometido fraude y el $34 \%$ otro tipo de mala conducta grave ${ }^{13}$. En general esto es considerado como la punta del iceberg porque muchos trabajos no pasan exámenes tan minuciosos.

La retracción es la acción de retirar un artículo de circulación, se publica y comunican los motivos por los que el artículo no debe ser citado ni mencionado como referencia. Lo cual es considerado como un acto muy vergonzoso para los autores y su institución, además de ser un trabajo adicional al proceso de edición. En octubre 2012, Fang et al. ${ }^{14}$ publicaron en PNAS un artículo que ha sido comentado y citado en varios periódicos y algunas revistas. Ellos analizaron poco más de 2000 artículos de la base de datos de PubMed y encontraron que el $43 \%$ fueron retractados por ser fraudes y el 14 y $10 \%$ fueron por publicación duplicada y plagiarismo respectivamente. Algunos investigadores pueden ver a la retracción como una penalidad inocua y sin importancia, sin embargo existen observadores como Retraction Watch (http://retractionwatch.wordpress.com) que es un blog donde se documentan estos casos ; y podemos mencionar el caso de Scott Reuben que tuvo varios trabajos retractados en la revista Anesthesiology News y que posteriormente fue enjuiciado por fraude de atención médica, condenado a seis meses de prisión federal y el pago de $\$ 415000$ en multas, decomiso y la restitución ${ }^{15}$.

Cokol $^{16}$ et al. ensayaron un modelo para tener una idea de cuantos trabajos deberían de ser retractados. Describe un modelo estocástico con parámetros que implican el rigor para aceptar una pequeña fracción de "manuscritos fallados" y dos factores sobre el escrutinio post-publicación en función del factor de impacto (IF). Entre sus conclusiones encuentra que las revistas con revisiones exigentes tanto las de alto IF como las de bajo IF presentarían menos artículos que podrían ser retractados. Además, en aquellos con más alto IF, la retracción es más severa en post-publicación. Aunque sus resultados relacionarían la eficiencia de la producción con las probabilidades de publicar artículos con mala conducta; también podríamos interpretar que los autores inescrupulosos identifican aquellas revistas que son más permisivas, y que no tienen filtros exigentes sobre los trabajos fraudulentos o con comportamientos de mala conducta. Evidentemente si se tratan de comportamientos volitivos, de los cuales se conocen sus consecuencias, tendremos un modelo donde la tendencia es a atiborrar con trabajos indeseables a las revistas con menos recursos. 


\section{Quiero publicar mi trabajo}

¿Algo de lo mencionado arriba sucederá en la Revista Peruana de Biología? La respuesta es sí. Por lo menos la llegada de trabajos fraudulentos y con malas conductas sí suceden y frecuentemente. Es una experiencia que se puede comprobar, si alguien desea obtener trabajos fraudulentos lo único que tiene que hacer es emprender una revista científica, llevar a cabo una revisión por pares, lograr visibilidad en bases de datos, ser más o menos constante y esperar, pronto llegaran.

¿Qué esta sucediendo? Simplemente que los editores de revistas "pequeñas" se enfrentan no solo a la problemática de la investigación nacional, a la falta de recursos, políticas de investigaciones débiles o inexistentes, sino al maremágnum de investigadores que quieren que sus investigaciones sean publicadas, generalmente presionados por compromisos de diversa índole y no porque ellos piensen que la información en su trabajo es relevante o contribuirá con la ciencia. Entonces, será común ver como son sometidos a la Rev peru biol los trabajos que fueron rechazados en otras revistas, y las que rechacemos irán a otras, en una cascada hasta que por fin lleguen a una revista donde serán publicados. La revista que se ubique al final de la cascada será una revista basurero.

Si bien es cierto en la Rev peru biol hemos recibido trabajos fabricados, plagios, auto-plagios, trabajos fragmentados; los más comunes son aquellos en que se omite información (consciente o inconscientemente). Algunas veces sobre el mismo trabajo, ocultando errores de muestreo o por ejemplo un experimento donde más de la mitad de individuos de prueba murieron por razones desconocidas antes de culminar el experimento. Otras omiten información sobre trabajos similares que deberían de discutirse, en algunos casos por no tener acceso a la publicación original, otras porque sus resultados no se pueden contrastar, mostrando fallas metodológicas, o por que pondrían en evidencia otro tipo de errores, incluso conceptuales. Otro tipo frecuente es la fragmentación de los trabajos, donde en lugar de brindar un trabajo completo que de luces a un problema, muestran parte por parte la información sin un trabajo de análisis y aporte significativo, así por ejemplo como si quisiéramos dar a conocer inventarios de ambos lados de una quebrada en sendos artículos. La publicación de todos estos tipos de trabajos solamente es contraproducente y dañina no solo para la revista sino para la institución y los científicos nacionales en general por el descredito que sufrirían nuestras investigaciones.

Este fenómeno debe ser enfrentado dentro de cada una de las revistas que se proponga llegar a la calidad que tanto esperan sus usuarios y también por las instituciones que apuestan (e invierten muchas veces sus escasos recursos) por realizar investigación científica como la UNMSM. Debe dedicarse considerable esfuerzo para no publicar trabajos fraudulentos, por los obvios motivos de mantener la reputación, la credibilidad, y el peso de nuestras ideas y propuestas en la sociedad. La realización de este esfuerzo disminuirá la llegada de esos tipos de trabajos. Pero también, debemos aplicar la censura post-publicación, y debe ser asumida por la institución y practicada por las revistas. Las retracciones deben de ser indicadores de nuestro esfuerzo de mejorar nuestra publicación, de lograr la credibilidad y calidad. La institución debe internalizar en sus políticas los aspectos de evaluación de calidad del trabajo de investigación y de las publicaciones y no solamente de los procedimientos y normas.

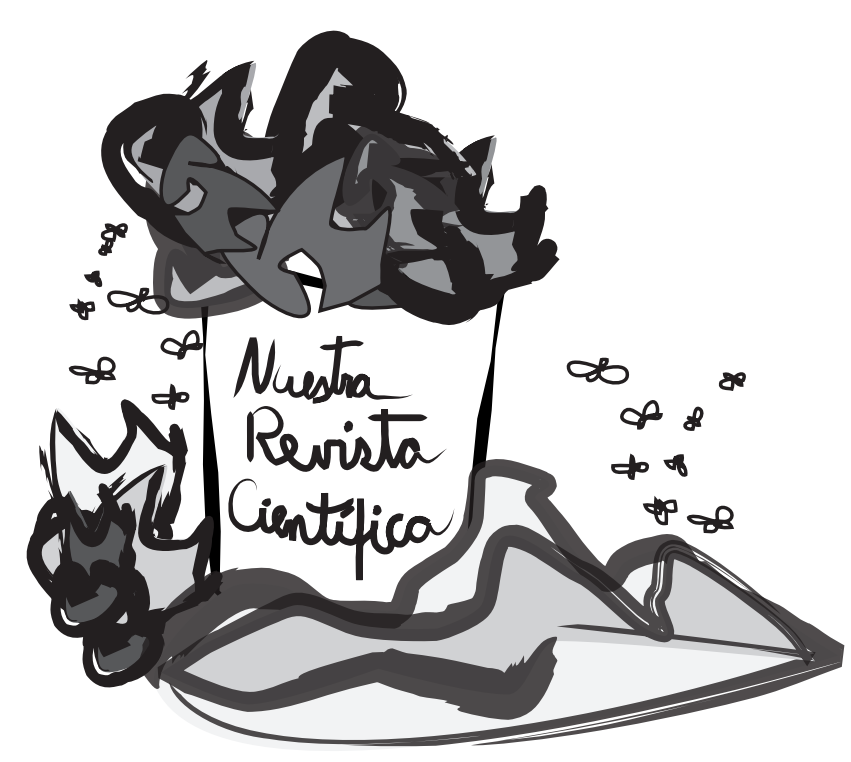

Una revista sola no podrá sobrevivir en este escenario, para ella el drama acabaría en tragedia; la trama empezaría cuando ella comience a incrementar su esfuerzo en prevenir el fraude, pronto la cantidad de artículos comenzará a disminuir (no es una revista fácil, pensaran los autores inescrupulosos), luego no podrá llegar a los estándares de publicación de las bases de datos más importantes (que piden más artículos por año) y luego se extinguirá lentamente. Para sobrevivir necesitará de una comunidad científica que la apoye, una comunidad crítica que tenga clara su misión con respecto de la ciencia y la sociedad. Necesitará de instituciones con políticas coherentes y robustas, que se fijen los objetivos a alcanzar.

\section{Referencias}

1 The Royal Society. 2011. Knowledge, networks and nations: Global scientific collaboration in the 21 st century. RS Policy document 03/11. London. http://royalsociety.org/policy/reports/knowledge-networks-nations.

2 Peña V. A., Rivera P., Schulz P.A.B. \& Tapia J.R.B. 2011. La Producción Científica en San Marcos. Hechos, cifras y estándares internacionales 2002-2010. Ediciones Vicerrectorado Académico UNMSM. CONCYTEC. 684pp.

3 Marusic A., V. Katavic \& M. Marusic. 2007. Role of editors and journals in detecting and preventing scientific misconduct: strengths, weaknesses, opportunities, and threats. Medicine and law 26 (3): 545-566.

4 Scott-Lichter D. \& the Editorial Policy Committee Council of Science Editors. 2012. CSE's White Paper on Promoting Integrity in Scientific Journal Publications, 2012 Update. 3rd Revised Edition. Wheat Ridge, CO: Available at: http://www.councilscienceeditors.org/files/public/ entire_whitepaper.pdf.

5 http://www.ithenticate.com/resources/overview/

5 http://www.crossref.org/crosscheck/index.html

6 DeMaria A.N. 2012. Scientific Misconduct, Retractions, and Errata. Journal of the American College of Cardiology 59 (16): 1488-1489. doi:10.1016/j.jacc.2012.03.005.

7 http://www.elsevier.com/about/publishing-guidelines/publishing-ethics

8 Franzen M., S. Rödder \& P. Weingart. 2007. Fraud: causes and culprits as perceived by science and the media. Institutional changes, rather than individual motivations, encourage misconduct. EMBO Reports 8 (1): 3-7. doi:10.1038/sj.embor.7400884.

9 Kreutzberg G.W. 2004. The rules of good science. EMBO reports 5 (4): 330-332. doi:10.1038/sj.embor.7400136. 
10 Publishing Research Consortium. 2008. Peer review: benefits, perceptions and alternatives. Mark Ware Consulting. http://www.publishingresearch. net/documents/PRCsummary4Warefinal.pdf

11 Peters D.P. \& S.J. Ceci. 1982. Peer-review practices of psychological journals: The fate of published articles. Behavioral and Brain Sciences 5 (2): 187-255. doi:10.1017/S0140525X00011183.

12 Butler, D. 2010. Journals step up plagiarism policing. Scientific American. http://www.scientificamerican.com/article.cfm?id=journals-policeplagiarism

13 Fanelli D. 2009. How Many Scientists Fabricate and Falsify Research? A Systematic Review and Meta-Analysis of Survey Data. PLoS ONE 4 (5): e5738. doi:10.1371/journal.pone.0005738.
14 Fang F.C., R.G. Steen \& A. Casadevall. 2012. Misconduct Accounts for the Majority of Retracted Scientific Publications. Proceedings of the National Academy of Sciences 109 (42): 17028-17033. doi:10.1073/ pnas. 1212247109 .

15 O'Reilly K.B. 2012. Misconduct accounts for most journal retractions. AMA. Amednews.com. http://www.ama-assn.org/amednews/2012/10/15/ prsb1015.htm

16 Cokol M., I. Iossifov, R. Rodriguez-Esteban \& A. Rzhetsky. 2007. How Many Scientific Papers Should Be Retracted? EMBO Reports 8 (5): 422-423. doi:10.1038/sj.embor.7400970. 